\title{
Research in Rocket and Jet Propulsion*
}

\author{
DR. HSUE-SHEN TSIEN \\ Robert H. Goddard Professor, California Institute of Technology
}

W HEN considering the problems of basic research in rocket and jet propulsion, it is profitable to keep in mind the salient features of rocket- and jet-propulsion engineering. These are: short duration of operation of the powerplant and extreme intensity of reaction in the motor.

That the duration of operation of the powerplant is short stems from the high specific consumption of the propellant. On the other hand, the dry weight of the rocket engine is much lower than that of other engines of equal output. Therefore, the total in. stallation weight (the sum of dry weight and propellant consumed) can be lower than other powerplants if the duration of operation is short. (See Ref. 1.)

Furthermore, the specific consumption of rocket engines at all speeds, and of ramjet engines at supersonic speeds, in terms of $l b / h r / l b s$ thrust, is essentially independent of flight speed. Therefore, the propulsive work done by the engine on the vehicle, per lb of fuel or propellant consumed, will be larger if the flight speed is larger. For this reason, it is advantageous to operate the rocket and the ramjet engine at large thrust and thus accelerate the vehicle to high speed.

The great kinetic energy of the vehicle at the end of the "burning time" of the powerplant is then utilized to achieve range by coasting. This form of dynamic trajectory is demonstrated to be superior to steady flight with long-drawn-out operation of the rocket and ramjet. Accordingly, all applications of these powerplants will involve intensive, but short-duration, operations of the engines.

The extreme intensity of reaction in the motor means high operating

\footnotetext{
* Condensed from a paper presented during the combined anntial conventions of the American Rocket Society and the American Society of Mechanical Engineers, Dec. 1, 1949, in New York.
}

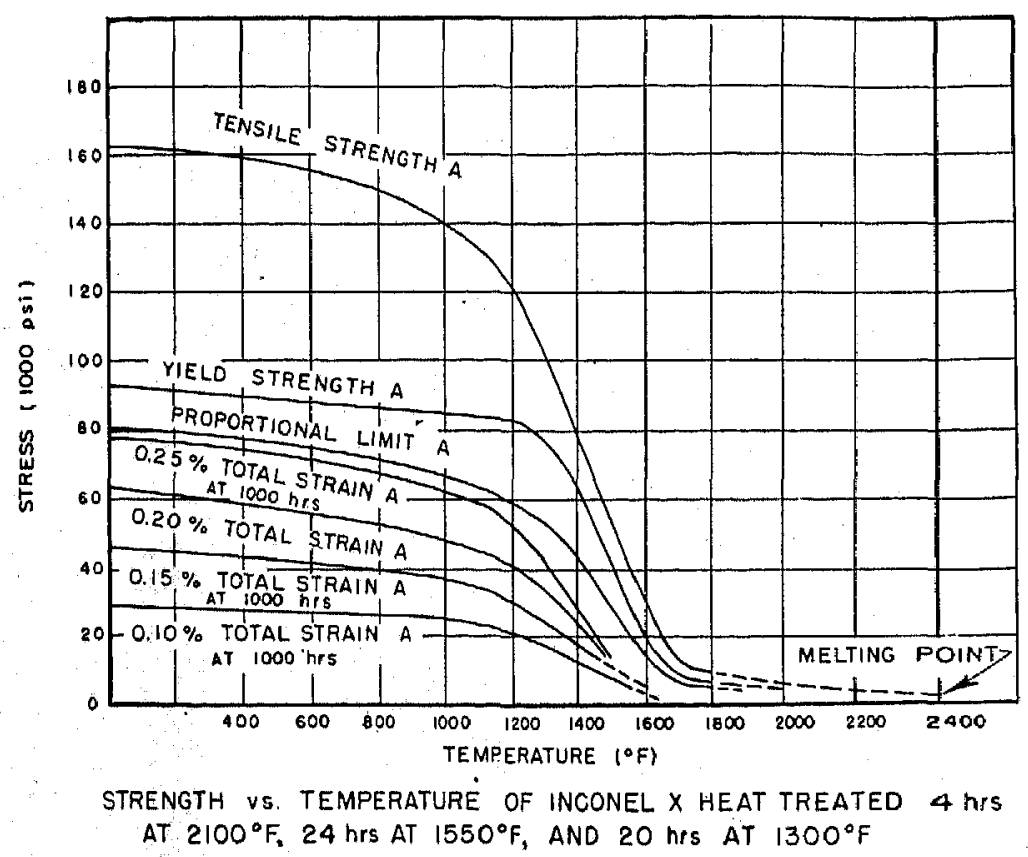

Figure 1

temperatures. To find materials which can withstand high stresses at high temperatures is the main material problem in rocket and jet-propulsion engineering. However, the problem here is different in one aspect from the material problem in turbojet and gas-turbine design. This is the short operating time of the unit. For expendable units, such as missiles, the operating time is generally of the order of minutes. Even for vehicles which are intended for repeated operation, it still is likely that the optimum performance will be obtained by a design which requires replacing the high-temperature and highly stressed parts after each operation.

By adopting this concept of designing for minutes instead of designing for thousands of hours as in the case of gas turbines, the material will be stressed for ultimate strength and not for creep. This difference is illustrated in fig. 1, where the stresses are plotted against temperature. The lower curves are design curves for creep, and the upper curves are the ultimate stress, a stress which is practically inde. pendent of the rate of strain.

For long operating time, the ultimate stress is not a design criterion, as the rate of strain near this stress is so large that the limiting strain will be reached long before the intended lifetime of the part, and the part will then fail. If the part is designed to have a life of only a few minutes, it can be stressed six times higher. This is a tremendous possibility in design and occurs only in rocket- and jetpropulsion engineering.

To explore this advantage leads, however, to complex problems in the stress and deflection analysis. The high rate of strain means constantly changing dimensions of the part, and its influence must be determined. The 
problem is not that of plasticity where the stress-strain relation is non-linear, nor that of elasticity, because now the material flows. In other words, the material must be considered as a visco-elastic medium.

As a first approximation, the stressstrain relation can be still considered as linear. To be specific, let $\sigma_{x}, \sigma_{y}$, $\sigma_{z}, \tau_{x y}, \tau_{y z}, \tau_{z x}$ be the six stress components, $\epsilon_{\mathrm{x}}, \epsilon_{\mathrm{y}}, \epsilon_{\mathrm{z}}, \gamma_{\mathrm{xy}}, \gamma_{y z}, \gamma_{\mathrm{zx}}$ be the six strain components. The stressstrain relation for isotropic, visco-elastic media can be written as

$$
\begin{aligned}
& \mathrm{p} \sigma_{\mathrm{x}}=\phi\left(\lambda \mathrm{e}+2 \mu \epsilon_{\mathrm{x}}\right) \\
& \mathrm{p} \sigma_{\mathrm{y}}=\phi\left(\mathrm{ye}+2 \mu \epsilon_{\mathrm{y}}\right) \\
& \mathrm{p} \sigma_{\mathrm{z}}=\phi\left(\lambda \mathrm{e}+2 \mu \epsilon_{\mathrm{z}}\right)
\end{aligned}
$$

$\mathrm{p} \tau_{\mathrm{xy}}=\phi \mu \gamma_{\mathrm{xy}}, \mathrm{p} \tau_{\mathrm{yz}}=\phi \mu \gamma_{\mathrm{yz}}, \mathrm{p} \tau_{\mathrm{zx}}=$ $\phi \mu \gamma_{\mathrm{zx}}$ where $\lambda$ and $\mu$ are constants and

$$
\mathrm{e}=\epsilon_{\mathrm{x}}+\epsilon_{\mathrm{y}}+\epsilon_{\mathrm{z}} \text {. }
$$

The operators $p$ and $\phi$ are linear time operators defined as:

$$
\begin{aligned}
P & =\frac{\partial \mathrm{m}}{\partial \mathrm{t}^{\mathrm{n}}}+\mathrm{a}_{\mathrm{m}-1} \frac{\partial \mathrm{m-1}}{\partial \mathrm{t}^{\mathrm{m}-1}}+\ldots \mathrm{a}_{0} \\
\Phi & =\frac{\partial^{\mathrm{n}}}{\partial \mathrm{t}^{\mathrm{n}}}+\mathrm{b}_{\mathrm{n}-1} \frac{\partial^{\mathrm{n}-1}}{\partial \mathrm{t}^{\mathrm{n}-1}}+\ldots \mathrm{b}_{0}
\end{aligned}
$$

The a's and b's define the property of the material. They could be functions of time, but not functions of the space variables. Thus, a material with changing properties, caused by the drift toward thermodynamic and chemical equilibrium, also can be represented by these operators.

\section{Variable Stress}

An analysis (Refs, 2 and 3) of the mechanics of such materials reveals that, if the load on the part is specified by a time factor $\mathrm{g}(\mathrm{t})$, then the stress distribution at any instant can be calculated as if the material is purely elastic with the same instantaneous load. The deflection of the structure is, of course, different. But it is specified by a time factor $h(t)$ which is independent of the particular value and distribution of the load and is only dependent on $g(t)$ and is determined by

$$
\mathrm{Qh}(\mathrm{t})=\mathrm{pg}(\mathrm{t})
$$

The $h(t)$ is thus a "universal" function in the sense that it is related only to $g(t)$ and the properties of the material. The other characteristics of the problem do not enter into its determination. In particular, the function $h(t)$ may be measured directly, experimentally, on a pure tension bar with the tension varied with time according to $\mathrm{g}(\mathrm{t})$. This is then a considerable simplification of the mechanics of visco-elastic media and a useful tool in the application of the idea of design for short-time flow of material.

The extreme intensity of reaction in

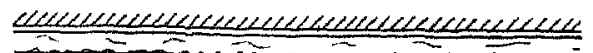
$\rightarrow \neg, \Omega, 7,7$ $\neg 7,7$ TURBULent CORE,

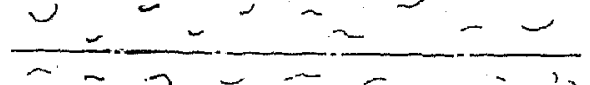

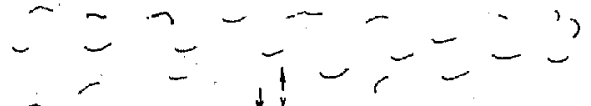

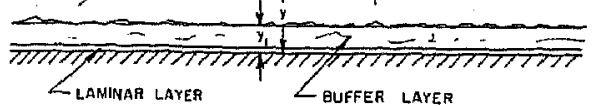
(1) TURGULENT CORE

$$
\frac{\underline{U}}{U_{1}}=1394+5.5 \log _{10}\left|y / y_{1}\right|
$$

(2) gUfFer layer and laMinar layeR

$$
\frac{r}{p}=v \frac{d u}{d y}-\overline{u^{\prime} v^{\prime}}+\frac{d \nu}{d T} \overline{T^{\prime}\left(\frac{\partial v^{\prime}}{\partial x}+\frac{d u^{\prime}}{\partial y}\right)}
$$

\section{Figure 2}

the rocket motor and in the combustion chambers of ramjets and pulsejets, and the high velocity of gas flow, lead to a very high rate of heat transfer to the walls. For instance, at the throat of a rocket nozzle, heat flux as high as 6 Btu per sec per $s q$ in has been observed. Changed into conventional units in other branches of engineering, this is more than 3 million $B t u$ per hour per $s q f t$. To cope with this high heat flux, designers have been forced to extrapolate the empirical laws of heat transfer to a cooling liquid and to seek other unconventional methods, such as surfaceboiling heat transfer.

\section{Heat Transfer}

To absorb the high heat flux by circulating a cooling liquid in a duct surrounding the hot chamber, one must use large differences between the wall temperature of the cooling liquid under turbulent-flow conditions. Here the problem is the lack of proper understanding of the basic mechanism. At present, the designer relies on empirical rules which are only safe to use within the range of variables of the test result.

To extrapolate without the guidance of a sound understanding of the phenomena is satisfactory. Of course, the problem of turbulent heat transfer has been attacked successfully by $O$. Rey. nolds, L. Prandtl, G. I. Taylor, Th. von Karmen and others. But their work is based upon the assumption that the temperature difference between the wall and the bulk of the

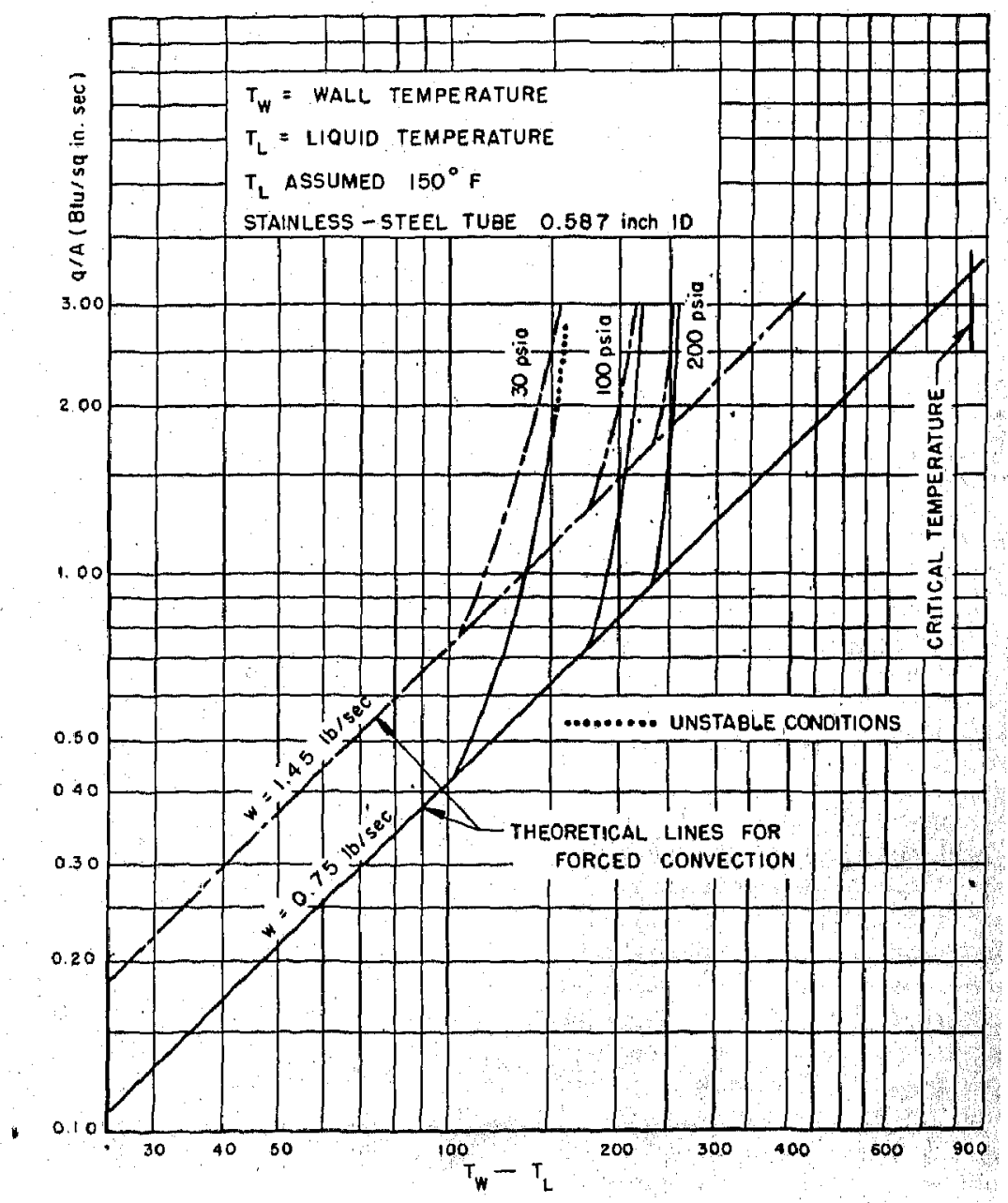

Figure 3. 


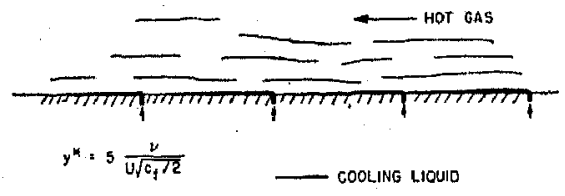

Figure 4

liquid is small, so that the flow is essentially isothermal.

Turbulent flow in a duct-say a circular pipe-can be divided into three regions (fig. 2): the turbulent central core where Reynolds' turbulent shearing stress dominates the molecular or viscous shearing stress, the laminar layer next to the wall where the viscous shearing stress dominates the turbulent shearing stress, and the buffer layer where both shearing stresses are important. For the turbulent central core, which occupies most of the pipe, previous experiments with isothermal flow indicate that the flow in general, and the velocity profile in particular, are controlled by the shear stress $\tau$ at the boundary of the turbulent core, the density $\rho$ of the liquid and a linear dimension $y_{1}$. Since the boundary of the turbulent core is very close to the wall, $\tau$ is practically equal to the wall shearing stress $\tau_{0}$. Together with $\rho, \tau_{0}$ can define a velocity $\mathrm{U}_{r}$ by

$$
\mathrm{U}_{\tau}^{2}=\frac{\tau_{0}}{\rho}
$$

Then, if $\mathrm{U}$ is the velocity at a point $y$ from the wall, the non-dimensional equation for the velocity profile must be

$$
\frac{\mathrm{U}}{\mathrm{U}_{\tau}}=\mathrm{f}\left(\mathrm{y} / \mathrm{y}_{1}\right)
$$

Clearly, the only available linear dimension for flow near the boundary of the turbulent core is the distance of this boundary from the wall. Hence, for flow near the boundary of the turbulent core, $y_{1}$ must be the thickness of the laminar and buffer layer. From previous experiments with isothermal flow in smooth pipes (Ref. 4 ), it is found that

$$
\frac{\mathrm{U}}{\mathrm{U}_{\tau}}=13.94+5.5 \log _{10} \frac{\mathrm{y}}{\mathrm{y}_{1}}
$$

Since temperature differences in the liquid will change only the viscosity, and, aceording to experiments viscosity does not enter directly into the turbulent core flow, the velocity relation given above must hold also for non-isothermal flow.

The problem now is to determine the thickness $y_{1}$. This thickness will vary with the temperature conditions. The work of H. Reichardt (Ref. 5) does not account for this variation and is therefore unsatisfactory. Thus, the main effect of higher temperature differences is in the buffer and the laminar layers. Here the variation of viscosity with temperature changes the flow. For instance, the effective shear stress $\tau$ is given by

$\underset{\rho}{\tau}=\nu \frac{\mathrm{dU}}{\mathrm{dy}}-\overline{\mathrm{u}^{\prime} \mathrm{v}^{\prime}}+\frac{\mathrm{d}}{\mathrm{dT}} \mathrm{T}^{\prime} \overline{\left(\frac{\partial \mathbf{v}^{\prime}}{\partial \mathrm{x}}+\frac{\partial \mathbf{u}^{\prime}}{\partial \mathrm{y}}\right)}$

where $\nu$ is the kinematic viscosity, $\mathrm{T}$ the temperature, $u^{\prime} v^{\prime}$ the instantaneous turbulent velocities in the directions parallel with the wall and normal to the wall, and $T^{\prime}$ is the temperature fluctuation. The bar over the second and third terms means averaging with respect to time.

The third term does not occur for isothermal flows. By its appearance in the equation for shear and the variable $v$ in the first term means now the effects of heat conduction, and the effects of shear are now coupled. The solution is thus more difficult than the corresponding isothermal problem, but the difficulty is believed to be surmountable.

When the wall temperature is raised beyond that of the boiling of the liquid under prevailing pressure in the pipe, local vaporization takes place and bubbles are formed over the surface. But, since the main bulk of the liquid is still at a temperature below the boiling point, these bubbles cannot grow indefinitely. In fact, experiments by F. Kreith and M. Summerfield show that they contract again and have a life span of about $1 / 100$ second. During its short life span, the bubble does not seem to move appreciably from the wall. The main consequence of the bubble formation and disappearance is the strong agitation of the fluid near the wall.

It is then understandable that the heat flux can be increased to many times that of the case without local boiling. This fact is shown clearly in fig. 3, taken from the work of Kreith and Summerfield (Refs, 6 and 7 ). This means that a high rate of cooling can be achieved without high flow velocity in the cooling duct. The thusreduced pressure drop in the cooling duct will decrease the necessary pumping work of the coolant. Boiling-heat transfer then can be used to good advantage for many designers. The problem for research here, of course, is a closer understanding of the turbulent

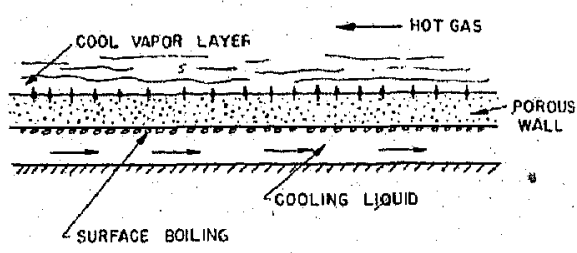

Figure 5 agitation due to bubble formation and thus better correlation of tests $f_{0}$ different liquids and different test conditions.

If the wall temperature is increased beyond a critical value over the boil. ing point of the liquid, it has been found that a vapor envelope forms over the surface, and the heat flux is reduced by the insulating effect of the stagnant vapor. Therefore, with speci. fied pressure and flow velocity, there is definitely a maximum value of heat. flux density even with local boiling at the surface. If still, high heat-flux density is desired, then other means of cooling have to be used.

\section{Wall-Temperature Effects}

However, even before reaching this intrinsic limit of boiling.heat transfer, the wall temperature at the inside sur. face of the rocket motor may be too high for the material strength, due to the necessary temperature gradient through the wall for the heat flux. For instance, if the heat flux is $6 \mathrm{Btu}$ per sec per $s q$ in, the wall thickness is $1 / 16$ in, and the temperature at the cool side of the wall is $600^{\circ} \mathrm{F}$, the temperature at the hot side of the wall will be $1950^{\circ} \mathrm{F}$ if stainless steel is used. This temperature is certainly too high for good strength. New, powerful cooling methods for extremely high heat flux are sweat-cooling and film-cooling.

Film cooling ( fig. 4) is achieved by establishing a thin liquid film in contact with the hot gas over the surface to be cooled. Due to the shearing stress acting over the liquid-gas interface, the liquid flows in the downstream direction. Simultaneously, the heating of the film by the hot gas evaporates the liquid. It is seen that, so long as there is a liquid film, the wall temperature is kept below the boiling point of the liquid.

It is noted also that, to protect the wall from the hot gas, liquid film has to be reestablished by injection through holes in the wall when the film upstream injection is evaporated. Of course, the intervals of injection can be lengthened by injecting more liquid and establishing a thicker film each time. However, the difficulty here is the instability of the film against the turbulent flow in the gaseous boundary layer. The resultant partial breakaway of the liquid in the form of droplets constitules a loss in effective cooling liquid. The problem here is then the determination of the rela tive cooling efficiency with respect to film thickness.

From experience on one-phase tur bulent boundary layer, it is found that the laminar sublayer thickness $y^{*}$ is determined. (Continued on page 124) 


$$
y^{*}=5 \frac{v}{U \sqrt{\frac{C_{l}}{2}}}
$$

where $v$ is the kinematic viscosity of the fluid, $U$ is the freestream velocity and $\mathrm{C}_{\mathrm{t}}$ is the local friction coefficient. If this relation holds also for twophase turbulent boundary layers such as exist in film cooling, $y^{*}$ is the limiting film thickness for perfect effciency. If the film thickness is larger than $y^{*}$, instability of the film and breakaway of droplets is likely to occur. It is then seen that there is an advantage in having a higher kinematic viscosity $v$, as layer thickness is allowed.

If $\mathrm{U}=1000 \mathrm{ft} / \mathrm{sec}, \mathrm{C}_{\mathrm{t}}=0.004$, and $y=0.319 \times 10^{-5} \mathrm{ft} / \mathrm{sec}$ for water at $212^{\circ} \mathrm{F}, \mathrm{y}^{*}$ is only $4.3 \times 10^{-6}$ in. This result indicates that, for theoretical maximum cooling efficiency, the film should be very thin and reestab. lished frequently along the wall. The limiting case is sweat-cooling, where the coolant is forced through the porous wall and injection and evaporation occur at the same time.

Sweat-cooling is, however, not lim. ited to the liquid coolant. The coolant may be gaseous. In fact, the most extensive experiments are made by $\mathrm{P}$. Duwez and H. L. Wheeler (Ref. 8) with gaseous coolants. However, it is shown by the above investigators that the coolant cannot be allowed to evaporate in the porous wall, as then the flow is essentially not stable, with wide fluctuations in the wall temperatures.

Generally, then, the most efficient sweat-cooling system, with least expenditures of the coolant, is one that evaporates the liquid coolant on the "outside" surtace of the porous wall before entering the porous material (fig. 5). In a sense, this system is a combination of boiling-heat transfer and sweat-cooling. No extensive experiment on this method of cooling has yet been made.

It is evident that, with either filmcooling or sweat-cooling, there is no limit to the temperature of the com. bustion gas that can be handled effectively. Therefore, one need have no misgivings about the high-energy fuels and propellants for cooling difficulties. Furthermore, for rocket, ramjet and pulsejet, there is no contact of the combustion gas with a delicate moving part, such as turbine blades in a turbojet; and the combustion gas can be corrosive and can contain finely, divided solid particles. These factors remove practically all restriction on the choice of fuels and propellants. Such strange combinations as liquid hydrogen and liquid fluorine, and diborane

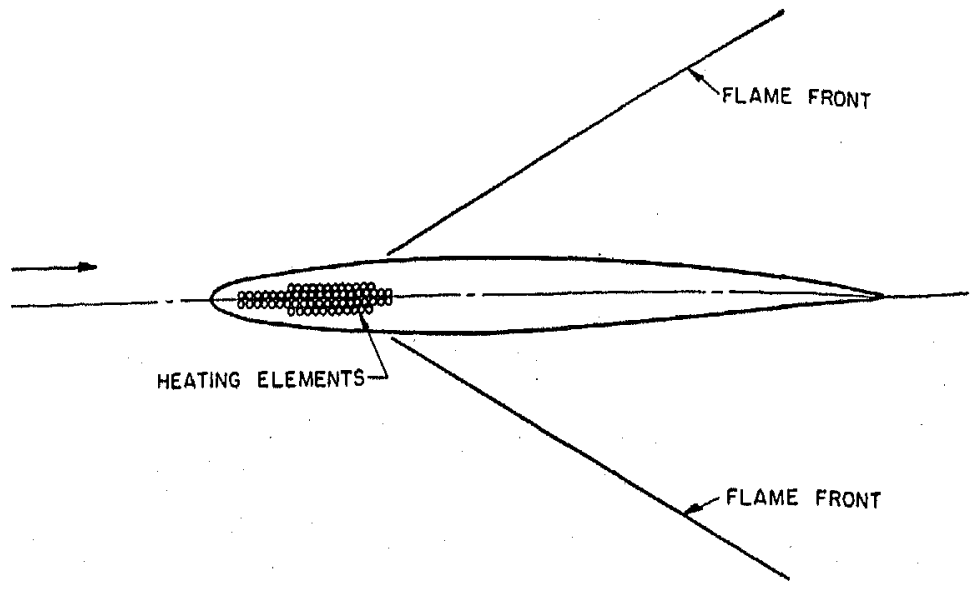

Figure 6

$\left(\mathrm{B}_{2} \mathrm{H}_{6}\right)$ and air, are to be considered. The more urgent problems of com. bustion in jet propulsion are those connected with fluid mechanical aspects. These are the auto-ignition of liquid jets, the evaporation of liquid droplets, the mixing of gaseous components, the mutual influence of combustion and turbulence of low combustion in the heterogeneous mixture etc. For ramjets, the most perplexing problem today is the problem of flame stabilization. This is a problem which confronts all ramjet designers. Worse still, the mechanism of flame stabilization is not yet understood. As a result, the flame-holder design for the combustion chamber is always done by ad hoc experimentation.

Clearly, there is a need for experiments with the simplest physical conditions, so that the parameters can be controlled. The work of A. S. Scurlock (Ref. 9) with homogeneous gas stream and controlled turbulence in the initial stream is the most noteworthy effort in this direction. How. ever, for a true understanding of the mechanism, further detailed explora. tion of flow field is necessary.

It seems that one of the important aspects of flame stabilization is the interaction of the flame front and the boundary layer. To test this concept, a flame holder is the shape of a streamlined body may be tried ( fig. 6). To start the combustion, the airfoil has to be heated first by, say, an electric current, to a high temperature. Once the flame is started, the airfoil aft of the flame is heated by the hot gas. Heat is then conducted through the body of the airfoil to the front section, where heat is given up to the cold gas mixture through the boundary layer. The cold gas mixture in the boundary layer being heated by the body will increase the concentra. tion of active carriers and finally ignite at the intersection of the flame front and the surface of the body.

It is evident that such a flame holder without turbulent and eddying loss has many practical applications despite the difficulty in starting. In fact, by increasing the length of the airfoil in the hot gas, the temperature at the front part can be increased, and thus the gas velocity can be raised without blowouts.

The ultimate aim of all this basic research is, of course, to improve the performance of rocket and jet-propelled vehicles. However, even - when given the best powerplant, the designer still has to determine the best way of using it for optimum performance of the completed vehicle. For instance, what would be the optimum thrust programming for a sound rocket? What would be the gain possible by varying the thrust during ascent? Is this gain justified by the additional complication in the design?

The basic variational problem of thrust programming was studied by G. Hamel (Ref. 10). However he made no detailed calculations to allow the designer to weigh the importance of different aspects of the problem.

\section{Long-Range Trajectory}

But the fundamental question in the performance analysis is the trajectory, particularly the long-range trajectory. Earlier in this discussion, the reason for favoring the dynamic trajectory of varying velocity was given. But what particular dynamic trajectory? To avoid the penalty of high drag at high velocity in the dense atmosphere and yet to be able to accelerate the vehicle quickly, it is clear that the vehicle should be launched vertically.

Performance of the vertical trajectory of a rocket ois well-known. But is a rocket the only powerplant capable of vertical trajectory? Certainly the ramjet-once boosted to a suff- 
ciently high velocity-also can produce enough thrust to make an accelecated vertical flight A ramjet would weigh more than a rocket per $l b$ of thrust: produced, but the fuel consumption is very much smaller. Preliminary estimates by Z. H. Schindel (Re) I. 1) show that the advantage of low fuel constumption ovcrcomes the disadvantage of heavier dry weigh:. Therefore, there is a definite gain in substituting the lowest stage of a multi-stage rocket with a ramjet. of course, to boost the ramjet to operating speed, it has to be operated as a ducted rocket in the first fow seconds.

What can one say about the rernainder of the trajectory? Since the high velocity of the vehicle is reached outsicle the atmosphere by vertical or near vertical ascent, the first part of the trajectory has to be liftless and this is elliptical. When the vehicle returns to the atmosphere at practically the same speed as it leaves the atmosphere, the lift of the body of the vehicle can be produced by putting the body into an angle of attack. The question kere then is one of programming the angle of attack of the body so that the maximum range is obtained.

As an example of such a dynamic tra. jectory, the flight of a 3000 -mile rocket vehicle is studied under the assumption of steady glide after the initial elliptical path. The average lift-drag ratio in glide is taken to be 4\% The result of this analysis is as follows:

Longth

$78.9 \mathrm{H}$

Maximum diametor

of body

$8.86 \mathrm{ft}$

Gross woight

Fuel load

$96,500 \mathrm{lbs}$

Weight after

"Brennschluss"

Propelfant load-

ing fraction

Exhaust velocity

Propellant

$72,400 /$ bs

$24,100 \mathrm{lbs}$

0.750

$12,000 \mathrm{ff} / \mathrm{sec}$.

liquid $\mathrm{O}_{2}$ liquid $\mathrm{H}_{3}$

liquid $\mathrm{F}_{2}$ liquid $\mathrm{H}_{2}$

Maximum valocity $9140 \mathrm{mph}$

Rango at conclu-

sion of olliptic

path

1200 miles

Range contributed

by glide

Altitude at ber

ginning of glide

Landing speed

Landing angle

1800 milos

27 miles

$50 \mathrm{mph}$

$20^{\circ}$

Flight duration Less than one haur

Thus it is seen that the requirements of a transcontinental rocket-liner are not at all beyond the grasp of present-day technology. The wings need not be large to achieve a teasonable landing speed, and the specifications on the structural weights are not impossible. When will such a rocket-liner be realized? That is a difficult question. But one thing is certain: the basic research as outlined in this discussion definitely will hasten the day of long-range rocket travel.

\section{REFERENCES}

1. A. Is. LowELL. "A Guide to Aircraft Powerplant Selection " Aeronautical Engineering Revisw, Vol, 6 , No. 4, pp. $22-25$ (1947)

2. T. ATFRHY. "Non-Fomogeneous Stresses in Visco-elastio Media," Querterly of Applied Mechanios, Vol. 2, pp. 113-119 (1944)

s. H. S. TsIEN, "A Generalization of Alfrey's theorem fo: Visco-elastic Media" Quarterty of Applied Mechantos (1950)

4. J. NIxuRaDse. "Gesetzm\&ssigkeiten der turbulonten Stromuting in glatten Rohren," Ver Deutsoh. Ing. Forschungshoft, No. 356 (1932)

5. FI, RaIchardT, "Die Walmetibertragung in turbulenten Relsbungschichten", Z.a.M.M. Vol. 20, pp. 297-328 (1940)

6. F. KRetTh and $M$. Summenfreld. "Heat Transfor to Water at High Flux Densities with and without surface BoilIng." ASME Transactions.

Also: "Investigations of IIeat Trangfer" at High Heat-Fiux Densities: Experimental Study with Water of Friction Drop and Forced Convecton with and without Surface Bolling in Tubes, Progress Report N. 4-68, Jet Propulsion Laboratory, CIT (1948)

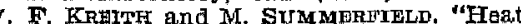
Transter from an inlectrically Heated Tube to Antine at High Heat Flux,"
Progress Report No. 4-88, Jet Propul sion Laboratory, CIT (1949)

8. $P$. DTtry and IF $Z$ W perimental study of Conling by Injection of a Elutd through a Forous Ma terial," $J$, of Aaro. Baimoes, Vol. 16, pp. $500-521$ (1948)

9. A C. SKURLook. "Flame Stabilization and Propagation in Higl-Velocity Ga Streams," Metoor Report No, 19 Mass. Inetitute of Technology (1948)

10. G. Fum "THber elne mit dem Prob. lem aer Rakete zusammenhangende Aufgabe der Veriationsrechnung, Z.a.M.M. Vol. 7, pp. 451-452 (1927)

11. $Z$. H. Scrimow: Application of IRamjet to High Attitude Sounding Vehtclos," M. $s$ rithesis, Dept. Aeronautlcal Eng neering, Mass. Inst. of Technology $(1047)$.

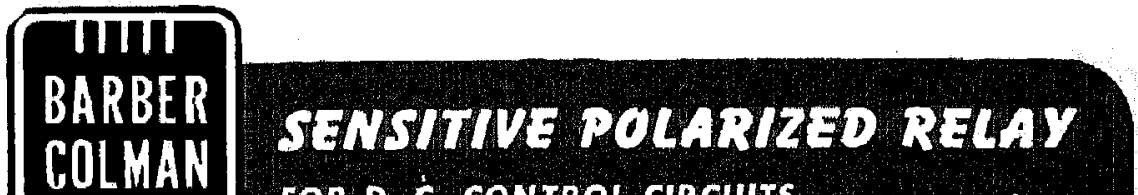

UIII

FOR D. C. GONTROL CIRCUITS

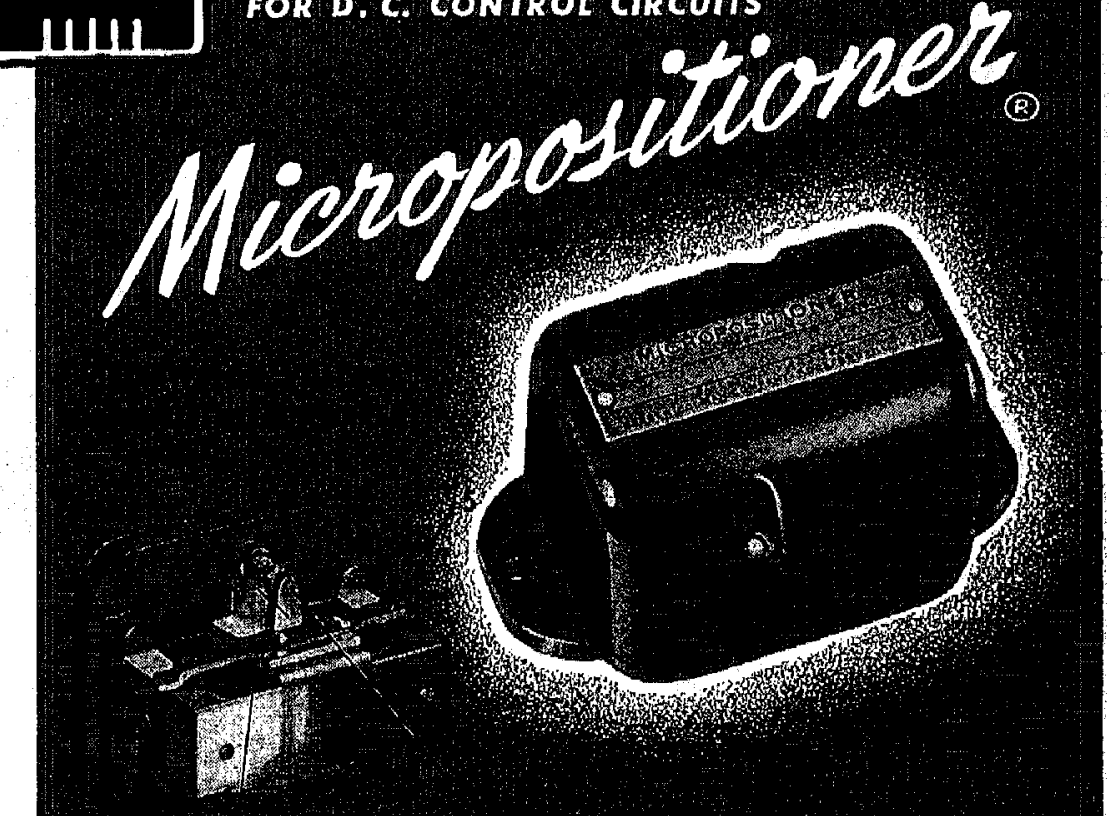

BARBER-COLMAN COMPANY - ROCKFORD, ILLINOIS 